\title{
(Psycho)therapies Primer and Selection Guide
}

\section{Different Patients, Different Therapies: Optimizing Treatment Using Differential Psychotherapeutics. Edited by Deborah L. Cabaniss and Yael Holoshitz; WW Norton \& Company; New York, New York; 2019; ISBN 9780393713428; pp. 512; \$ 59.95 (hardcover).}

\author{
Richard Balon ${ }^{1}$
}

Received: 28 February 2020 / Accepted: 24 March 2020 / Published online: 13 April 2020

(C) Academic Psychiatry 2020

At the completion of the initial evaluation, many, if not most, psychiatrists who feel psychotherapy is indicated tell the patient something like, "You need therapy." Is that enough to say, though? Should the recommendation be more specific and informative, similar to the recommendation of a specific medication? Do psychiatrists actually know enough to make such a specific recommendation for therapies beyond those which are mandated in resident training? Myriad psychotherapeutic options are available, and some of them have been proven quite effective.

Two teachers of psychotherapy at Columbia University, Deborah Cabaniss, M.D., and Yael Holoshitz, M.D., gathered a large group of experts in various psychotherapies and put together a book that intends to help "operationalize how we think about and use multiple psychotherapeutic frameworks" (p xxi) to make an informed decision about what psychotherapy to recommend.

The book consists of three parts: Part I, Differential Psychotherapeutics, written by the editors, introduces the concept of differential psychotherapeutics (i.e., the practice of thinking about patients using multiple therapy frameworks) in chapters focused on four "iterative, collaborative steps: learning, thinking, matching, discussing" (p. 3). The editors emphasize the following:

Therapists have to do more than just think about their own framework: they have to have at least some sense of other frameworks and treatments so they can optimize care for the patients who consult them. This is what differential psychotherapeutics (DP) is all about. It

Richard Balon

rbalon@wayne.edu

1 Wayne State University, Detroit, MI, USA means approaching patients with the idea that there are multiple ways to think about and treat patients. It requires therapists to abandon the idea that there is only one right way, and instead to think about how to optimize treatment for this person, in this place, at this time. We can call this the DP stance. Different therapies serve different purposes for different patients. ...The DP stance also asks therapist to allow themselves to remain agnostic about what they will recommend until they have learned about the patient, know something about the patient's internal and external resources, and have a good sense of the patient's goals. (pp. 4, 5)

Part II, Psychotherapeutic Options, again written by the editors, clusters in five chapters 23 different therapies on the basis of their "theoretical and technical commonalities" (p. 41), including (1) cognitive and behavioral therapies (CBT; e.g., CBT for psychosis, prolonged exposure therapy, exposure and response prevention therapy, schema therapy, dialectical behavior therapy, eye movement desensitization, and reprocessing); (2) psychodynamic and supportive psychotherapies (e.g., transference-focused psychotherapy, panicfocused psychodynamic psychotherapy, brief dynamic psychotherapy, psychoanalysis, and supportive psychotherapy); (3) interpersonal psychotherapies (e.g., complicated grief therapy); (4) family therapies (e.g., couples therapy and parentchild interaction therapy); and (5) value-based and strengthbased therapies (e.g., acceptance and commitment therapy, motivational interviewing, and solution-focused brief therapy).

These five chapters have a similar structure, using the framework provided in Part I, discussing the background of each modality and then going through learning, thinking, and matching and the typical treatment trajectory and common techniques. A great summary table of therapies and their main features is included at the end of Part II. 
Part III, Differential Psychotherapeutics in Practice, consists of 12 brief, fictional patient histories in various clinical situations (e.g., substance use, depression, parenting, psychosis, trauma, affect dysregulation, and low self-esteem). Each patient's story is followed by three write-ups from experts in various psychotherapies (38 writers). The experts used the differential psychotherapeutics rubric outline, discussing what they learned by reading the case, what they thought about it, how they would match the patient to the therapy modality, and how they would discuss the treatment in a session (p. 127). Each selected psychotherapy write-up starts with a box that lists recommendations of what to listen for within the framework of this specific treatment modality. For example, those staring couples therapy for parenting issues should listen for individual and multigenerational family history particularly related to relationships and how symptoms and behaviors manifest in the context of family or couple. As the editor's note, it is important to realize "not all experts using a single treatment modality listen for exactly the same things, nor do they all use only one framework. In fact, many of our experts use several, often including a psychiatric or diagnostic framework" (p. 128). Examples of modalities selected for a specific case include selection of dialectical behavioral therapy; acceptance and commitment therapy; and motivational interviewing for substance use or supportive therapy, complicated grief therapy, and family therapy for late-life depression and grief.

The book also includes an 'Educators' Guide for Teaching Differential Psychotherapeutics" and guidance on "Using What You've Learned" with a worksheet for writing out the basic elements (e.g., learning and thinking). In the Endnote, the editors emphasize that differential psychotherapeutics is a lifelong practice rather than something to master easily.

I agree that there are limitations of recommending some therapies reviewed in this volume. As Drs. Cabaniss and
Holoshitz write, "It's difficult to recommend a psychotherapeutic modality if there isn't a therapist nearby who can conduct it. And it's hard to recommend a treatment that a patient can't afford or doesn't have time to pursue" (p. 4). The editors also did not include some modalities that one may consider important, namely group therapy and psychoeducation alone (mentioned as part of several therapies). As an educator, I would also welcome an expanded guide for educators, applicable to residency training. Some of these issues may be addressed in the next edition of this volume.

Nevertheless, differential psychotherapeutics should be something we all ultimately should be practicing, and the psychotherapies reviewed in this book should become a part of the psychiatric armamentarium. As noted, psychiatrists have to be able to have at least some sense of other treatments and therapies frameworks and be able to optimize patient care by recommending them. We need to change teaching psychotherapy in our training programs accordingly by teaching more therapies and frameworks and by the way we determine which therapy fits which patient. It is a monumental task and a long-term goal, but it has to be done. This book is a great start. It should be read by all interested in psychotherapy education, program directors, psychotherapists, and clinicians who refer patients for psychotherapy.

\section{Compliance with Ethical Standards}

Disclosure The author declares no conflicts of interest.

Publisher's Note Springer Nature remains neutral with regard to jurisdictional claims in published maps and institutional affiliations. 